\title{
Equation of State and Freezeout in QCD with Staggered Quarks
}

\author{
Saumen Datta ${ }^{1}$, R. V. Gavai ${ }^{1}$, and Sourendu Gupta ${ }^{1, \star}$ \\ ${ }^{1}$ Department of Theoretical Physics, Tata Institute of Fundamental Research, Homi Bhabha Road, Mumbai \\ 400005, India
}

\begin{abstract}
We report the equation of state at finite chemical potential, namely the baryon number density and the baryonic contribution to the pressure, using a resummation of the Taylor expansion. We also report the freezeout conditions for a measure of fluctuations. We examine the major sources of systematic and statistical errors in all of these measurements.
\end{abstract}

\section{Introduction}

Currently our best estimate of the location of the critical point in 2 flavour QCD with approximately $230 \mathrm{MeV}$ pions and $N_{t}=8$ is

$$
\mu_{B}^{E}=(1.85 \pm 0.04) T^{E}, \quad T^{E}=(0.94 \pm 0.01) T_{c}
$$

where $T_{c}$ is the cross over temperature at $\mu=0$ [1]. It was noticed earlier that the position of the critical point is mildly dependent on the pion mass [2]. The continuum extrapolated 1- $\sigma$ lower bound of [3] lies just outside the 1- $\sigma$ upper bound of this result. The two are consistent at fairly high significance although the computations of [3] use 2+1 flavours of quarks with physical masses and are extrapolated to the continuum. This remarkably happy convergence of results means that one must start to look at physics beyond just the location of the critical point. Here we report a computation of the equation of state, and a non-equilibrium phenomenon called freezeout, using the configurations which gave the above estimate.

\section{Equation of state}

At a critical point the equation of state will have singularities which show up as a divergence in the baryon number susceptility (BNS),

$$
\chi_{B}^{2} \propto\left[\mu_{B}^{2}-\left(\mu_{B}^{E}\right)^{2}\right]^{-\psi}+\text { non - singular }
$$

This singularity is converted to a pole using the so-called DLOG

$$
m_{1}=\frac{\partial \log \left(\chi_{B}^{2} / T^{2}\right)}{\partial\left(\mu_{B} / T\right)}=\frac{2 \psi \mu_{B} T}{\mu_{B}^{2}-\left(\mu_{B}^{E}\right)^{2}} .
$$

^ Speaker, e-mail: sgupta@tifr.res.in 
Next, the Taylor series expansion for $\chi_{B}^{2}$ can be converted formally to one for $m_{1}$. Then the expression above gives a Padé approximant for the transformed series. Two terms can be used to extract the critical chemical potential and the critical exponent. All other terms serve as checks [1].

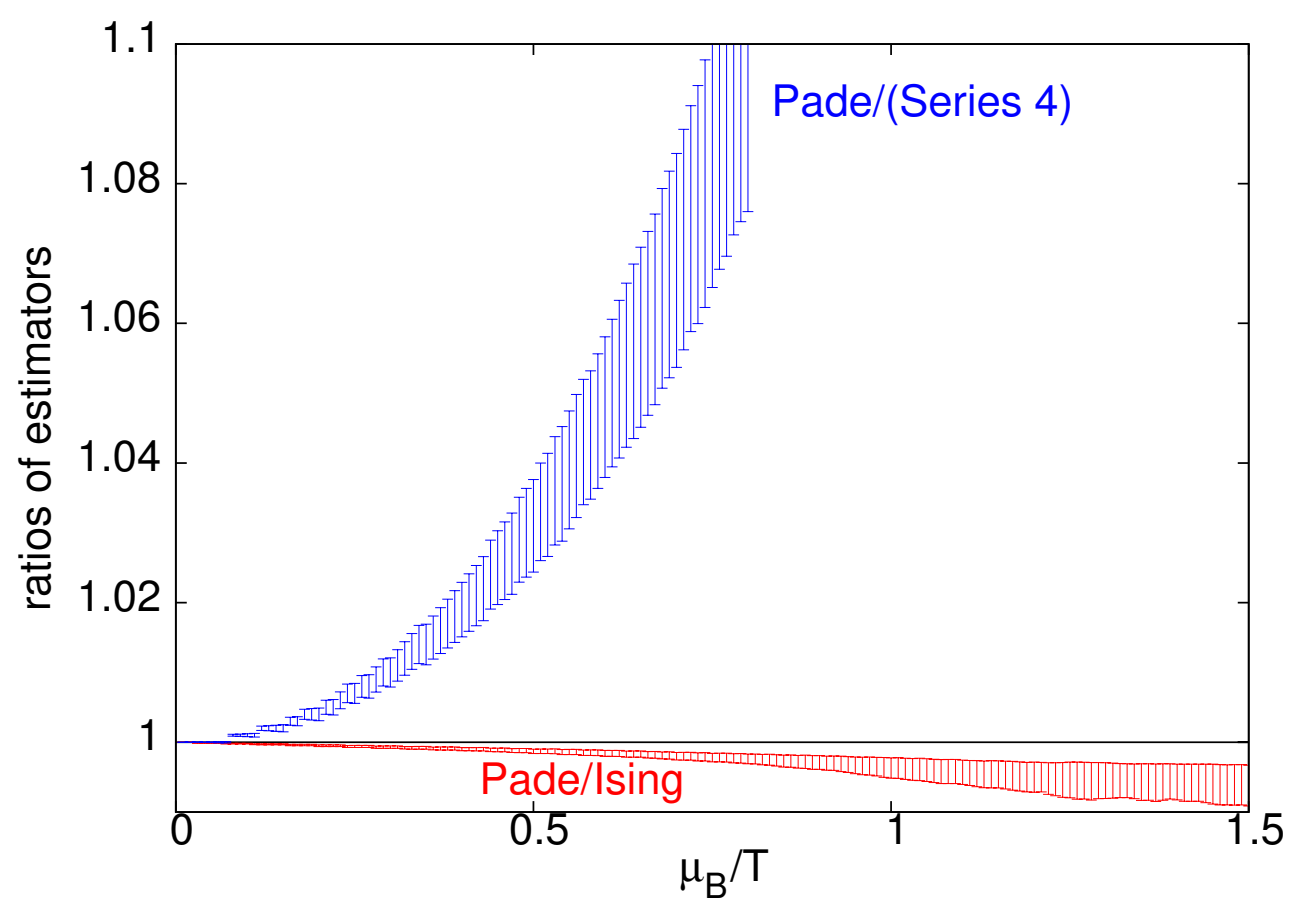

Figure 1. Comparing $\chi_{B}^{2}$ obtained by the Padé method (labelled Pade in the figure) with the series expansion truncated to two terms (labelled Series 4), and also the Padé method where $\psi$ has been fixed to its Ising value (labelled Ising). Notice that the Ising result is close to the unconstrained Padé result, indicating that the Ising critical exponent may be compatible with the computation. However, the Padé and the series differ from each other already at very small $\mu_{B}$. The bands are due to statistical errors.

The BNS can be obtained by integrating the Padé approximant and exponentiating the result (in this paper we call it the Padé method for the BNS). This gives the correct singularity to $\chi_{B}^{2}$. The alternative method of truncating the series expansion of $\chi_{B}^{2}$ [3] fails long before $\mu_{B}^{E}$. We show this in Figure 1. Series truncation is therefore the major source of systematic errors in the measurement.

The baryon number density, $n$, and pressure change, $\Delta P\left(T, \mu_{B}\right)=P\left(T, \mu_{B}\right)-P(T, 0)$, follow from $\chi_{B}^{2}$ by successive integrations. The resulting singularities in these two quantities are too mild to be seen by eye. The results for the equation of state as a function of temperature is shown in Figure 2 , for several values of the chemical potential. Our estimation of statistical errors takes into account autocorrelations. At such quark masses very close to the cross over, autocorrelations are very large, and they show up as large statistical errors in the figure. 

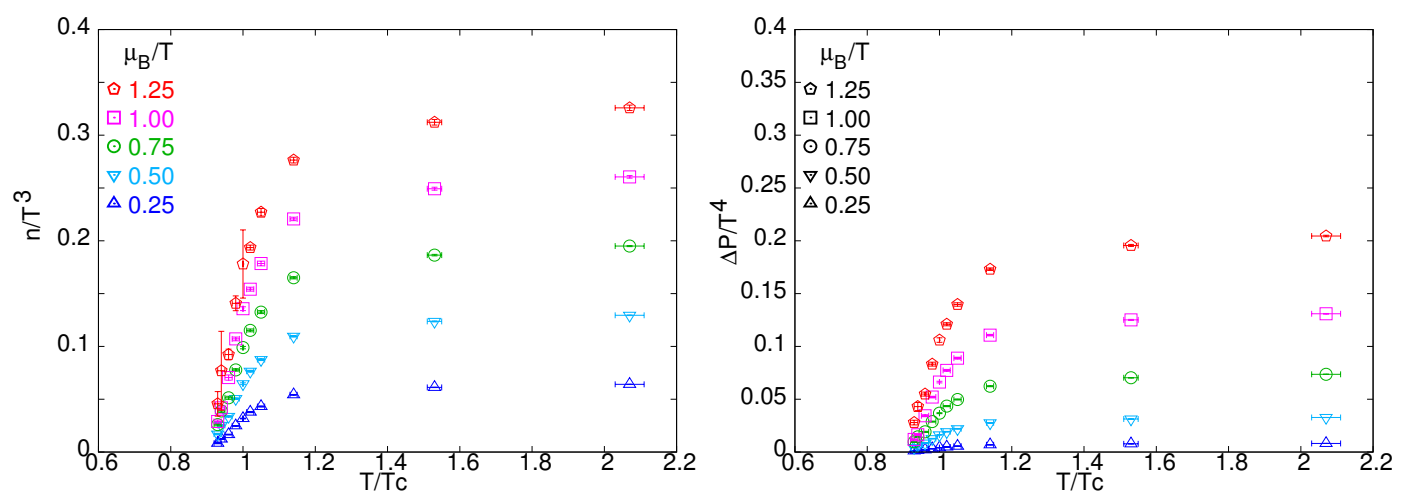

Figure 2. The equation state of two flavour QCD, obtained by successive integration of $m_{1}$.

\section{Freezeout}

The quantity $m_{1}$ can be written in a different way,

$$
m_{1}=\frac{\partial \log \left(\chi_{B}^{2} / T^{2}\right)}{\partial\left(\mu_{B} / T\right)}=\frac{\chi_{B}^{3} / T}{\chi_{B}^{2} / T^{2}} .
$$

In this form it was suggested as a quantity that could be measured in experiments first in $[4,5]$. The first experimental results were made in [6]. Later lattice computations of this quantity were reported in $[7,8]$.

Freezeout in heavy-ion collisions is a non-equilibrium process, and its comparison with equilibrium lattice computation involves the assumption that the system is in thermal equilibrium throughout its history, right until freezeout. Then a comparison of any measured quantity with thermal equilibrium computations would reveal the freezeout temperature and chemical potentials. The assumption is very likely to be somewhat wrong, since finite transport coefficients would smear out the freezeout. As a result, different quantities would show different freezeout conditions. Therefore, it is important for lattice computations to look at freezeout without input assumptions.

In studying the freezeout of fluctuations, one would like to discover at least two conditions: freezeout $T$ and $\mu_{B}$. One measurement, such as of $m_{1}$ cannot predict both. In [7], therefore, it is assumed that freezeout occurs at $T_{c}$. Freezeout of hadron yields do seem to occur near $T_{c}$ in some treatments [9], but it is a model dependent statement. Other freezeout models see different results [10-12]. It is safe to use $m_{1}$ to designate a region of $T$ and $\mu_{B}$ and ask whether other measurements give overlapping regions.

Comparing our computations with the measurements of the STAR experiment at their highest energy [6], we find the band of allowed $T$ and $\mu_{B}$ shown in Figure 3. The width of the band is dominated by the statistical errors from the lattice computation. Detailed studies of the systematic errors made if the same computation is done using a truncated series expansion $[7,13]$ are shown in [1]. Here we note a point which may have an interesting physics consequence.

To leading order in the expansion in $\mu_{B} / T$, one finds that the freezeout area from $m_{1}$ is linear in the $\mu_{B}-T$ plane. This is the reason that much attention has focussed on it. However, the full Padé resummation, shown in Figure 3, reveals that this is not true beyond the leading order. In fact, it appears that the increase in $T$ is very strongly tempered as $\mu_{B}$ increases, nearly reaching a ceiling at 


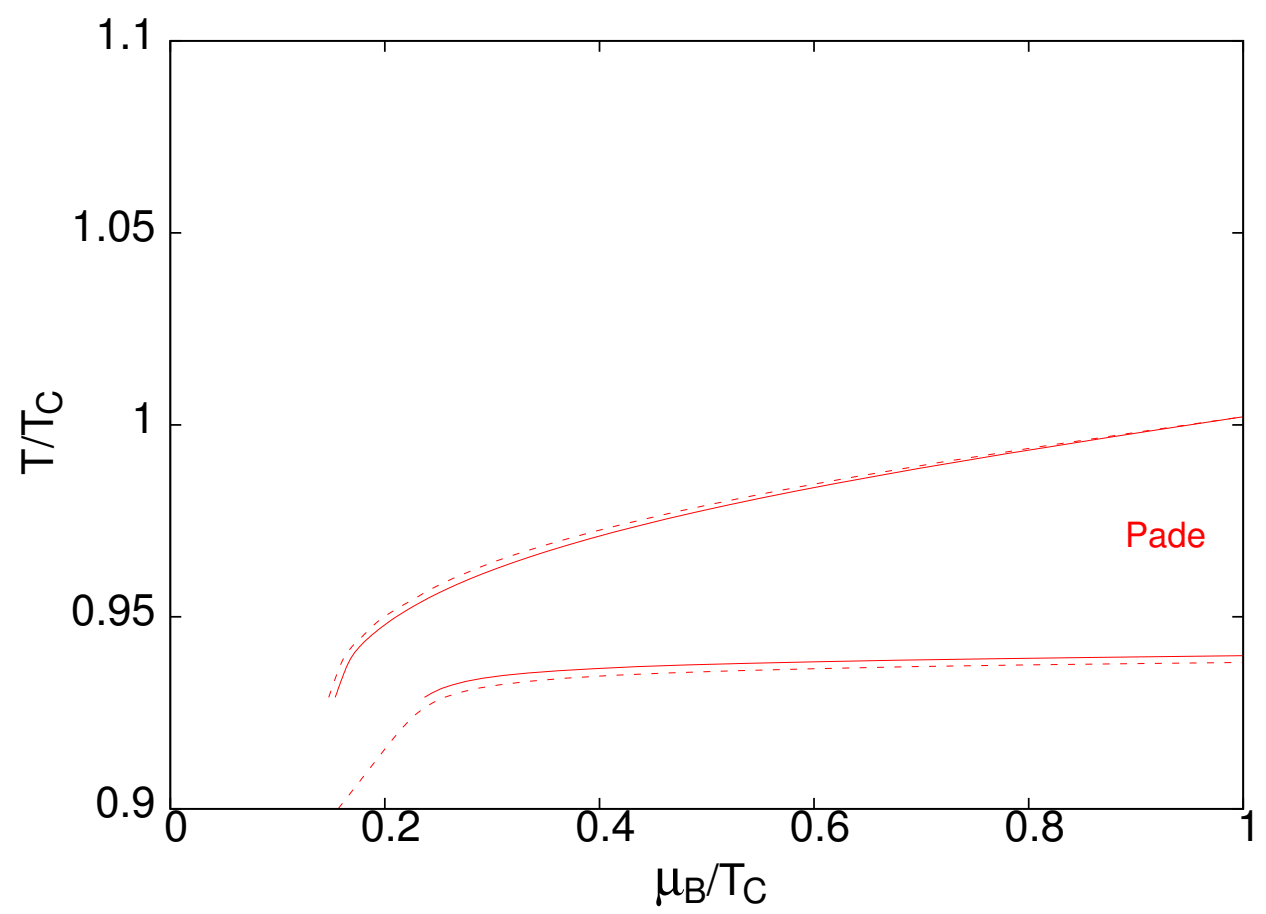

Figure 3. Freezeout conditions in STAR top energy collisions obtained by comparing our results for $m_{1}$ with STAR measurements. The width of the band is due to statistical errors in lattice measurements. The extra contribution to this uncertainty from experimental errors is shown as a dotted line.

$T_{c}$. This is an interesting new observation of what could happen if the heavy-ion fireball evolves close to equilibrium.

These computations were performed using the facilities of the Indian Lattice Gauge Theory Initiative (ILGTI) and the Department of Theoretical Physics, TIFR. 


\section{References}

[1] S. Datta, R.V. Gavai, S. Gupta, Phys. Rev. D95, 054512 (2017), 1612.06673

[2] S. Gupta, N. Karthik, P. Majumdar, Phys. Rev. D90, 034001 (2014), 1405.2206

[3] A. Bazavov et al., Phys. Rev. D95, 054504 (2017), 1701.04325

[4] S. Gupta, PoS CPOD2009, 025 (2009), 0909. 4630

[5] R.V. Gavai, S. Gupta, Phys. Lett. B696, 459 (2011), 1001.3796

[6] M.M. Aggarwal et al. (STAR), Phys. Rev. Lett. 105, 022302 (2010), 1004 . 4959

[7] A. Bazavov et al., Phys. Rev. Lett. 109, 192302 (2012), 1208.1220

[8] S. Borsanyi, Z. Fodor, S.D. Katz, S. Krieg, C. Ratti, K.K. Szabo, Phys. Rev. Lett. 111, 062005 (2013), 1305.5161

[9] J. Stachel, A. Andronic, P. Braun-Munzinger, K. Redlich, J. Phys. Conf. Ser. 509, 012019 (2014), 1311.4662

[10] F. Becattini, M. Bleicher, T. Kollegger, T. Schuster, J. Steinheimer, R. Stock, Phys. Rev. Lett. 111, 082302 (2013), 1212.2431

[11] S. Chatterjee, R.M. Godbole, S. Gupta, Phys. Lett. B727, 554 (2013), 1306.2006

[12] V. Begun, W. Florkowski, M. Rybczynski, Phys. Rev. C90, 014906 (2014), 1312 . 1487

[13] S. Gupta, Central Eur. J. Phys. 10, 1261 (2012), 1205. 1713 